\title{
Perioperative covert stroke in patients undergoing coronary artery bypass graft surgery
}

Austin Browne, PhD, ${ }^{a}$ Jessica Spence, MD,${ }^{\mathrm{a}, \mathrm{b}}$ Patricia Power, MSc, ${ }^{\mathrm{a}}$ Ingrid Copland, CCRA, ${ }^{\mathrm{a}}$ Rajibul Mian, PhD, ${ }^{\mathrm{c}}$ Stephanie Gagnon, BHSc, ${ }^{\mathrm{a}}$ Shauna Kennedy, MD, ${ }^{\mathrm{d}}$ Mukul Sharma, MD, ${ }^{\mathrm{a}, \mathrm{e}}$ and André Lamy, MD ${ }^{\mathrm{a}, \mathrm{f}}$

\section{ABSTRACT}

Objectives: Covert stroke is a complication of coronary artery bypass graft surgery that is increasingly recognized as a serious problem. In noncardiac surgery settings, covert stroke is associated with the development of delirium, long-term cognitive decline, and future clinical stroke. Therefore, we sought to determine the feasibility of conducting a large, prospective cohort study of the influence of covert stroke on neurocognitive outcomes in patients undergoing coronary artery bypass graft surgery.

Methods: NeuroVISION Cardiac pilot was a prospective cohort study enrolling patients aged $\geq 21$ years undergoing isolated coronary artery bypass graft surgery to receive diffusion-weighted magnetic resonance imaging of the brain after surgery to identify patients with covert stroke. Patients were screened for postoperative delirium in-hospital and were administered questionnaires of cognitive and global function (once before and twice after surgery). Regional cerebral oxygen saturation was recorded during surgery using near-infrared spectroscopy.

Results: Between March 27, 2017, and February 11, 2018, 50 of 66 patients enrolled (76\%) completed the brain magnetic resonance imaging (1 patient per week). Among the 49 patients included in the analysis, 19 (39\%; $95 \%$ confidence interval, $26 \%-53 \%)$ experienced perioperative covert stroke and $3(6 \%)$ had a clinical stroke within 30 days of surgery. Postoperative delirium occurred in $5(26 \%)$ patients with covert stroke and in $3(10 \%)$ patients who did not experience covert stroke.

Conclusions: The NeuroVISION Cardiac pilot study established the feasibility of conducting a large, prospective cohort study of the determinants and consequences of covert stroke in patients undergoing coronary artery bypass graft surgery. (JTCVS Open 2020;4:1-11)

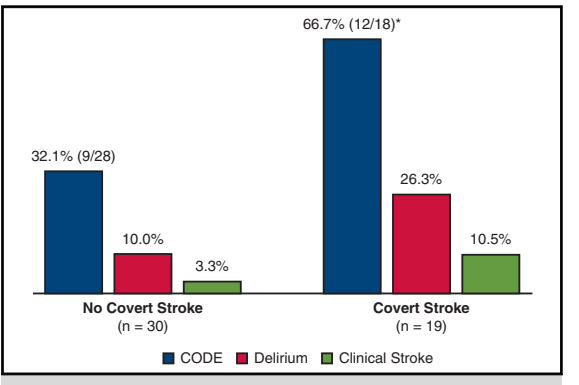

Incidence of neurologic complications in patients who experienced or did not experience covert stroke.

\section{CENTRAL MESSAGE}

This pilot study established the feasibility of conducting a large, prospective cohort study of the determinants and consequences of covert stroke in patients undergoing cardiac surgery.

\section{PERSPECTIVE}

In cardiac surgery settings, we know little about the determinants and consequences of perioperative covert stroke. The NeuroVISION Cardiac pilot study demonstrates the feasibility of conducting a large, prospective cohort study of the influence of covert stroke on delirium, longterm cognitive decline, and future clinical stroke in patients undergoing cardiac surgery.

See Commentaries on pages 12 and 14 .
Video clip is available online.

From the Departments of ${ }^{a}$ Perioperative Medicine and ${ }^{\mathrm{c}}$ Statistics, Population Health Research Institute, Hamilton, Ontario, Canada; and Departments of ${ }^{\mathrm{b}}$ Anesthesia, ${ }^{\mathrm{d}}$ Radiology, ${ }^{\mathrm{e}}$ Neurology, and ${ }^{\mathrm{f}}$ Surgery, Hamilton General Hospital, Hamilton, Ontario, Canada.

Funded by grants to Dr Lamy from the McMaster Surgical Association and scholarships to Dr Spence from the McMaster Regional Medical Association.

Received for publication Aug 17, 2020; revisions received Aug 17, 2020; accepted for publication Aug 18, 2020; available ahead of print Sept 17, 2020.
Coronary-artery bypass grafting $(\mathrm{CABG})$ is a commonly performed surgical procedure that improves clinical outcomes in patients with severe coronary artery disease. ${ }^{1}$ A key

Address for reprints: André Lamy, MD, Department of Perioperative Medicine, Population Health Research Institute, DBCVSRI Room C1-112, 20 Copeland Ave, Hamilton, Ontario L8L 2X2 Canada (E-mail: lamya@mcmaster.ca). 2666-2736

Copyright $(C) 2020$ The Authors. Published by Elsevier Inc. on behalf of The American Association for Thoracic Surgery. This is an open access article under the CC BY-NCND license (http://creativecommons.org/licenses/by-nc-nd/4.0/).

https://doi.org/10.1016/j.xjon.2020.08.008 


\section{Abbreviations and Acronyms \\ $\mathrm{CABG}=$ coronary artery bypass grafting \\ DSST $=$ Digit Symbol Substitution Test \\ DW-MRI = diffusion-weighted magnetic resonance imaging \\ MoCA = Montreal Cognitive Assessment \\ SAGE = Standard Assessment of Global Activities in the Elderly}

limitation of CABG surgery is neurological complications such as clinical strokes, covert strokes, short-term delirium, and long-term cognitive decline. ${ }^{2-6}$ Despite efforts to reduce neurologic complications (eg, precannulation assessment of aortic atherosclerosis, off-pump CABG, anaortic off-pump CABG, clampless proximal aortic devices, cerebral perfusion techniques, and cerebral oxygen saturation monitoring) clinical strokes, covert strokes, delirium, and cognitive decline are still among the most frequent and feared complications.

Covert strokes are recent small brain infarcts detected on brain imaging that are not associated with symptoms of clinical (overt) stroke. Diffusion-weighted magnetic resonance imaging (DW-MRI) allows for the detection of acute brain infarcts occurring up to 10 days before imaging; thus, it is not necessary to obtain a preoperative MRI scan to diagnose perioperative covert stroke. Although patients with covert strokes may present with no obvious symptoms, their presence is associated with the development of delirium, ${ }^{7}$ cognitive decline, ${ }^{7,8}$ dementia, ${ }^{5,8}$ and a 2 - to 3-fold higher risk of future clinical stroke., ${ }^{7,-12}$

Cardiac surgery patients are at elevated risk for developing cerebral injury due to their baseline comorbidities and the procedural risks of cardiac surgery. ${ }^{13}$ Like clinical stroke, atherosclerotic emboli liberated by surgical manipulation of the aorta most likely represents a key modifiable determinant of covert stroke. ${ }^{14}$ The prevalence of covert stroke in CABG surgery is estimated to be $25 \%$ (95\% confidence interval [CI], 15\%-35\%), ${ }^{15}$ more than 3-fold higher than patients undergoing noncardiac surgery $\left(7 \% ; 95 \%\right.$ CI, 6\%-9\%). ${ }^{7}$ A 2019 international study (Detection and Neurological Impact of Cerebrovascular Events in Noncardiac Surgery Patients: A Cohort Evaluation [NeuroVISION]) of 1114 noncardiac surgery patients using DW-MRI demonstrated that perioperative covert stroke is associated with an increased risk of delirium within 3 days and cognitive decline 1 year after surgery. ${ }^{7}$ In addition, a 2019 systematic review has outlined an important trend-the prevalence of covert strokes is increased in cardiac procedures with increasing levels of aortic manipulation ${ }^{15}$ - suggesting that aortic manipulation is a contributing mechanism for perioperative covert stroke. However, no large study has established associations between perioperative covert stroke and delirium, long-term cognitive decline, or the degree of aortic manipulation in patients undergoing cardiac surgery.

Brain oximetry is increasingly used during cardiac surgery to noninvasively monitor regional cerebral oxygen saturation to ensure adequate cerebral perfusion. ${ }^{16}$ Few studies have combined brain oximetry recordings with DW-MRI screening and therefore we aimed to clarify whether covert stroke is associated with acute declines in regional cerebral oxygen saturation during CABG surgery.

We conducted the NeuroVISION Cardiac pilot study to determine the feasibility of undertaking a large multicenter cohort study to clearly established an association between covert stoke and long-term cognitive decline and to determine whether surgical techniques that aim to reduce or avoid aortic manipulation can reduce rates of covert stroke.

\section{MATERIALS AND METHODS Study Design and Participants}

The NeuroVISION Cardiac pilot study was a prospective cohort study of 66 patients undergoing cardiac surgery conducted at the Hamilton General Hospital from March 2017 to February 2018. Patients were eligible to participate if they were aged $\geq 21$ years, scheduled to undergo isolated CABG surgery through a median sternotomy and had at least 1 of the following risk factors: peripheral vascular disease, cerebrovascular disease, renal insufficiency, diabetes mellitus, urgent CABG surgery, recent smoker, or left ventricular ejection fraction $<35 \%$. Patients were not eligible if they had a previous or emergency CABG surgery, planned circulatory arrest during surgery, dementia of any kind, or contraindication for DW-MRI.

The study was approved by the Hamilton Integrated Research Ethics Board before patient recruitment and registered with ClinicalTrials.gov (identifier NCT04241289). The funding agencies had no role in data interpretation and all participants provided written informed consent before completing any study-specific tasks.

\section{Data Collection}

Before surgery, trained research staff collected demographic and clinical information, assessed baseline cognitive function using the standardized Montreal Cognitive Assessment (MoCA) ${ }^{17}$ and Digit Symbol Substitution Test (DSST) ${ }^{18,19}$ questionnaires, and assessed global function using the Standard Assessment of Global Activities in the Elderly (SAGE). A description of each test is provided (Appendix 1). On the day of surgery, bilateral oximetry probes were positioned on the forehead before induction of supplemental oxygen and operative brain oxygenation was continuously recorded using the INVOS 5100C monitoring device (Covidien/Medtronic, Mansfield, Mass). A list of participating surgeons (Appendix Table 1) and study personnel (Appendix Table 2) are provided. Patients were screened for delirium twice daily in-hospital using the standardized Confusion Assessment Method ${ }^{20}$ starting the second day after surgery and ending after 3 days of negative tests. Cognitive assessments were administered in-person at discharge and 30 days after surgery. The SAGE was administered in-person at 30 days and by telephone at 90 days. The incidence of adverse clinical events (ie, death, stroke, myocardial infarction, and new renal failure requiring dialysis) were assessed at 30 and 90 days. Clinical outcomes were adjudicated by an expert clinician independent of study design and conduct. 


\section{Brain MRI}

Brain MRI were scheduled between 3 and 9 days after surgery. The MRI sequences included fluid-attenuated inversion-recovery, gradient-recalled echocardiogram, T2, and DW-MRI with apparent diffusion coefficient mapping. A Siemens 1.5 Tesla machine (Siemens Healthineers AG, Erlanger, Germany) was used and resulting images of cerebral infarcts were subclassified into acute ( $<24$ hours) and subacute ( 24 hours to 5 days) periods. Covert strokes were defined based on DW-MRI evidence of acute or subacute brain infarcts and the absence of a clinical stroke diagnosis following the brain MRI. The patients, outcome assessors, and clinical team were blinded to the results of the brain MRI to minimize potential evaluation bias. An expert in neuroradiology interpreted each brain MRI for acute infarction, chronic infarction, and incidental clinical findings (eg, tumors).

\section{Brain Oximetry}

In the operating room, a trained research coordinator applied 2 adhesive oximeter pads bilaterally over the frontotemporal area before induction of anesthesia. The sensors recorded regional cerebral oxygen saturation values for the duration of surgery with the output monitor covered to ensure that all members of the operative and research teams were blinded to the oximetry transcript to minimize potential treatment bias. During analysis of oximetry transcripts, baseline regional cerebral oxygen saturation measurements were determined for each patient, taken to be the mean of 20 recorded values between 25 and 15 minutes before cardiopulmonary bypass (ie, after the administration of supplemental oxygen by nasal cannula). We defined cerebral oxygen desaturation events as any regional cerebral oxygen saturation values $\geq 20 \%$ below baseline in either left or right or both hemispheres during surgery.

\section{Outcomes}

The primary outcome of the single-center NeuroVISION Cardiac pilot study was to determine the feasibility of conducting a larger multicenter study of covert stroke in patients undergoing CABG surgery. Feasibility was assessed by determining the overall recruitment rate at our center, the rate of brain MRI completion, and the proportion of patients lost to follow-up. Secondary outcomes included the incidence of covert stroke, delirium, intraoperative cerebral oxygen desaturation events based on oximetry transcripts, cognitive decline at discharge and 30-day follow-up based on MoCA and DSST, global function at 30- and 90-day follow-up based on SAGE, and major adverse cardiovascular events (composite of death, myocardial infarction, clinical stroke, and new renal failure requiring dialysis).

\section{Statistical Analysis}

Enrollment of a small series of patients at our center was a convenient sampling method to demonstrate feasibility. The primary analysis was per protocol and included only patients who completed the brain MRI. Patients who did not complete the MRI, completed the MRI but had results that could not be interpreted, or who were diagnosed with clinical stroke between the time of surgery and their MRI were excluded. The secondary analyses compared outcomes of the patients who had (covert stroke group) and who did not have (no covert stroke) a perioperative covert stroke at preoperative baseline, hospital discharge, 30 days, and 90 days after surgery. Analyses of cognitive and global function outcomes included only patients who completed the baseline assessment. Patients not completing assessments at subsequent time points were omitted from comparisons between time points. Changes in cognitive function from baseline were assessed using differences in test scores and clinical thresholds (ie, decrease of $\geq 2$ points on the MoCA and decrease of $\geq 5$ points on the DSST) used in recent studies. ${ }^{7,21}$ Analyses of oximetry data included only patients with complete intraoperative data who underwent surgery with the use of cardiopulmonary bypass. We report continuous variables using mean \pm standard deviation (SD) or median (interquartile range [IQR]) after testing for normality and binary or categorical variables as counts and proportions. Overall recruitment and feasibility characteristics are reported for all study participants. All statistical analyses were performed using SAS software version 9.4 (SAS Institute, Cary, NC).

\section{RESULTS \\ Feasibility Outcomes}

A total of 375 participants were screened for eligibility and 66 participants were enrolled over a 48 -week period for an overall recruitment rate of 1.4 patients per week (Figure 1). Fifty $(76 \%)$ of these patients completed the brain DW-MRI resulting in a pragmatic recruitment rate of 1 patient per week. The 16 patients without MRI results were excluded from analyses. The most common reasons for not completing the brain DW-MRI were patient refusal (8 $[12 \%]$ patients) or contraindication for MRI (5 [8\%] patients). A single patient with uninterpretable MRI results was excluded from the analyses. No participants were lost to follow-up at the final 90-day visit.

\section{Baseline and Operative Data}

Following the brain MRI study, 19 patients (39\%; 95\% CI, 26\%-53\%) had DW-MRI evidence of perioperative covert stroke in comparison to 30 patients $(61 \% ; 95 \%$ CI, 47\%-73\%) who did not experience a perioperative covert stroke (Table 1). Baseline characteristics were similar between patients with or without covert stroke. The mean \pm SD age of study participants was $67 \pm 9.3$ years; $42(86 \%)$ were men; mean European System for Cardiac Operative Risk Evaluation was $4.8 \pm 2.3$ points; $43(88 \%)$ had hypertension; $20(41 \%)$ had diabetes; and $2(4 \%)$ had a history of stroke.

Operative characteristics were also similar between patients with or without covert stroke. Overall, the mean \pm SD duration of cardiopulmonary bypass and crossclamping were $105.9 \pm 26.8$ and $79.9 \pm 29.0$, respectively; patients received $3.8 \pm 0.7$ grafts each; and $48(98 \%)$ patients underwent on-pump CABG surgery.

\section{Cognitive and Global Function Assessments}

Among all patients, mean $\pm \mathrm{SD}$ or median (IQR) preoperative baseline testing scores were $24.2 \pm 3.1$ on the MoCA; $42.9 \pm 13.2$ on the DSST; and 2.0 (IQR, 0.0-3.5) on the SAGE (Table 2). No differences in mean baseline scores between groups were found in either MoCA or DSST. However, baseline global function, as measured by SAGE, was superior (ie, lower scores) in patients who had a covert stroke compared with patients who did not have a covert stroke $(0.5 ;$ IQR, $0.0-2.0$ vs 2.0; IQR, 0.0-5.0; $P=.02$ ). No differences in MoCA or SAGE assessment completion rates were found between groups (Table 3). However, fewer patients completed the 


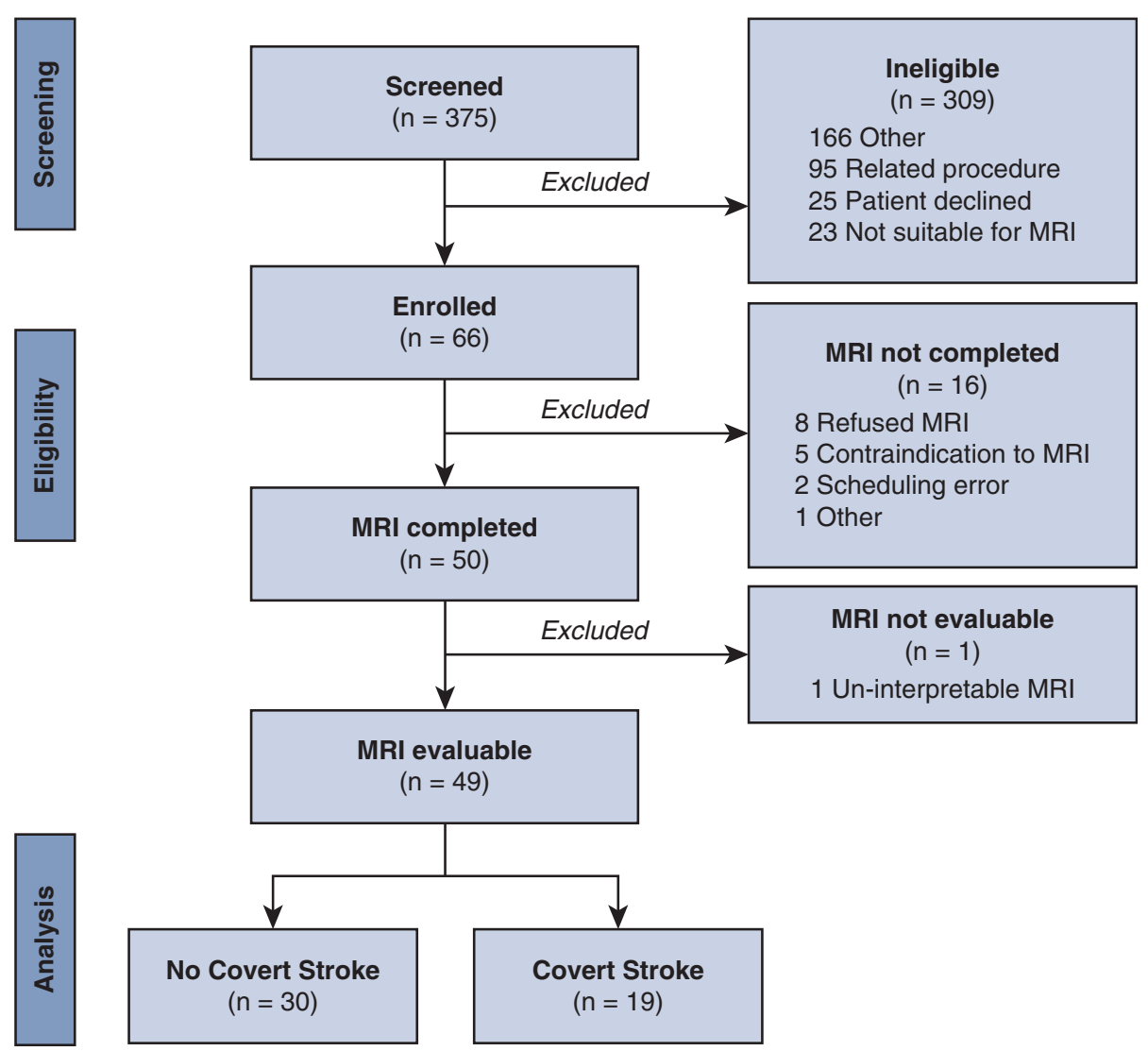

FIGURE 1. Distribution of patients in the pilot study with breakdown of reasons for exclusion. The most common reason for not completing the magnetic resonance imaging $(M R I)$ was patient refusal. Forty-nine patients with evaluable MRI results were included in the final analysis, of whom 19 experienced a covert stroke. Covert strokes were defined based on diffusion-weighted MRI evidence of acute or subacute brain infarcts and the absence of a clinical stroke diagnosis following the brain MRI.

DSST at both baseline and discharge visits in patients who had a covert stroke compared with those who did not have a covert stroke (13 [68\%] vs $29[97 \%] ; P=.006)$.

Changes in mean MoCA and DSST scores from preoperative baseline to discharge after surgery were similar between groups (Table 2). There was a small decline in cognitive function, as measured by the DSST, in patients who had a covert stroke $(-0.2 \pm 7.2[1 \%$ decline $])$ that was not observed in patients who did not have a covert stroke $(3.6 \pm 11.4[15 \%$ increase $])$ at the time of discharge, but this was followed by improvements in cognitive function in both groups at 30 days after surgery. Increases in cognitive test scores from baseline to 30 days were greater in patients with covert stoke compared with without covert stroke on the MoCA $(1.8 \pm 2.9[7 \%$ increase] vs $1.2 \pm 2.5$ $[5 \%$ increase $] ; P=.003)$ and DSST $(13.4 \pm 10.5[56 \%$ increase] vs $11.8 \pm 11.8$ [ $27 \%$ increase]; $P<.001)$. Lastly, there was a lack of improvement in global function, as measured by SAGE (ie, negative changes correspond to improved global function), in patients who had a covert stroke that were not seen in patients who did not have a covert stroke at 30 days $(0.0 ; \mathrm{IQR},-1.0$ to $1.0[0 \%$ improvement $]$ vs $-2.0 ; \quad \mathrm{IQR}, \quad-3.0$ to $0.0 \quad[100 \%$ improvement]; $P=.20$ ) and 90 days after surgery $(0.0$; IQR, -2.0 to $0.0[0 \%$ improvement $]$ vs -0.5 ( -3.5 to $0.0)$ [ $25 \%$ improvement]; $P=.73$ ).

In addition to changes in mean test scores of the cohort, we also identified patients with clinically relevant declines in cognition function (ie, cognitive decline) from baseline to 30 days after surgery. Cognitive decline, defined as a decrease of $\geq 2$ points on the MoCA, occurred in $1(8 \%)$ of 13 patients who had a covert stroke and in $2(8 \%)$ of 24 patients who did not have a covert stroke. Cognitive decline, defined as a decrease of $\geq 5$ points on the DSST, occurred in a single patient who did not experience covert stroke.

\section{Clinical Outcomes and Adverse Events}

Postoperative delirium occurred in 8 of 49 patients (16\%; $95 \%$ CI, 8\%-29\%). Among patients with delirium, 5 $(26 \%)$ had a covert stroke compared to $3(10 \%)$ who did not have a covert stroke (Figure 2 and Table 4).

Twenty-one of 46 patients ( $46 \%$; $95 \%$ CI, 32\%-60\%) had cerebral oxygen desaturation events during surgery. 
TABLE 1. Baseline and operative characteristics

\begin{tabular}{|c|c|c|c|c|}
\hline Characteristic & Overall $(N=49)$ & No covert stroke $(\mathbf{n}=\mathbf{3 0})$ & Covert stroke $(n=19)$ & $P$ value \\
\hline Age (y) & $67.1 \pm 9.3$ & $65.5 \pm 9.1$ & $69.6 \pm 9.4$ & .14 \\
\hline Male sex & $42(85.7)$ & $26(86.7)$ & $16(84.2)$ & .81 \\
\hline EuroSCORE & $4.8 \pm 2.3$ & $4.7 \pm 2.7$ & $5.0 \pm 1.6$ & .59 \\
\hline \multicolumn{5}{|l|}{ History of } \\
\hline Hypertension & $43(87.8)$ & $26(86.7)$ & $17(89.5)$ & .77 \\
\hline Myocardial infarction & $29(59.2)$ & $19(63.3)$ & $10(52.6)$ & .56 \\
\hline Diabetes & $20(40.8)$ & $10(33.3)$ & $10(52.6)$ & .24 \\
\hline Sleep apnea & $11(22.4)$ & $7(23.3)$ & $4(21.1)$ & .85 \\
\hline Current tobacco use & $9(18.4)$ & $6(20.0)$ & $3(15.8)$ & .71 \\
\hline COPD & $5(10.2)$ & $5(16.7)$ & $0(0.0)$ & .14 \\
\hline Atrial fibrillation & $3(6.1)$ & $2(6.7)$ & $1(5.3)$ & .84 \\
\hline Stroke & $2(4.1)$ & $2(6.7)$ & $0(0.0)$ & .51 \\
\hline Transient ischemic attack & $1(2.0)$ & $0(0.0)$ & $1(5.3)$ & .39 \\
\hline Family history of dementia & $0(0.0)$ & $0(0.0)$ & $0(0.0)$ & - \\
\hline \multicolumn{5}{|l|}{ Intraoperative data } \\
\hline CPB time (min) & $105.9 \pm 26.8$ & $103.4 \pm 27.2$ & $110.0 \pm 26.5$ & .41 \\
\hline Crossclamp time (min) & $79.9 \pm 29.0$ & $78.8 \pm 28.6$ & $81.9 \pm 30.3$ & .73 \\
\hline No. of grafts per patient & $4.0(4.0-4.0)$ & $4.0(4.0-4.0)$ & $4.0(3.0-4.0)$ & .96 \\
\hline On-pump CABG* & $48(98.0)$ & $30(100)$ & $18(94.7)$ & .39 \\
\hline Urgent surgery & $46(93.9)$ & 29 (96.7) & $17(89.5)$ & .55 \\
\hline Aortic calcification $\dagger$ & $4(8.2)$ & $3(10.0)$ & $1(5.3)$ & .56 \\
\hline
\end{tabular}

Values are presented as mean \pm standard deviation, $\mathrm{n}(\%)$, or median (quartile 1-quartile 3) unless otherwise indicated. EuroSCORE, European System for Cardiac Operative Risk Evaluation; $C O P D$, chronic obstructive pulmonary disease; $C P B$, cardiopulmonary bypass; $C A B G$, coronary artery bypass surgery. *A single patient in the covert stroke group underwent off-pump CABG surgery. $\dagger$ Moderate or severe aortic calcification.

Covert stroke was associated with higher rates of cerebral oxygen desaturation events during surgery (12 [67\%] patients with covert stroke vs 9 [32\%] without covert stroke; $P=.02$ ) (Figure 2 and Table 4 ).
Between MRI and 30-day follow-up, $3(6 \%)$ patients had clinical stroke; $2(11 \%)$ with covert stroke, and 1 (3\%) without covert stroke (Figure 2 and Table 4). One death occurred within 90 days of surgery in a patient

TABLE 2. Cognitive and global function assessments

\begin{tabular}{|c|c|c|c|c|}
\hline Assessment & Overall $(N=49)$ & No covert stroke $(n=30)$ & Covert stroke $(n=19)$ & $P$ value \\
\hline \multicolumn{5}{|l|}{ MoCA* } \\
\hline Baseline & $24.2 \pm 3.1$ & $24.2 \pm 3.0$ & $24.1 \pm 3.3$ & .95 \\
\hline \multicolumn{5}{|c|}{ Change from baseline } \\
\hline To discharge & $0.5 \pm 2.4(2)$ & $0.6 \pm 2.1(3)$ & $0.4 \pm 3.0(2)$ & .19 \\
\hline To $30 \mathrm{~d}$ & $1.4 \pm 2.6(6)$ & $1.2 \pm 2.5(5)$ & $1.8 \pm 2.9(7)$ & .003 \\
\hline Cognitive decline & $3 / 37(8.1)$ & $2 / 24(8.3)$ & $1 / 13(7.7)$ & .95 \\
\hline \multicolumn{5}{|l|}{ DSST $*^{*}$} \\
\hline Baseline & $42.9 \pm 13.2$ & $44.0 \pm 12.8$ & $41.1 \pm 14.1$ & .48 \\
\hline \multicolumn{5}{|c|}{ Change from baseline } \\
\hline To discharge & $2.4 \pm 10.3(6)$ & $3.6 \pm 11.4(15)$ & $-0.2 \pm 7.2(-1)$ & .14 \\
\hline To $30 \mathrm{~d}$ & $12.4 \pm 11.3(29)$ & $11.8 \pm 11.8(27)$ & $13.4 \pm 10.5(56)$ & $<.001$ \\
\hline Cognitive decline & $1 / 38(2.6)$ & $1 / 25(4.0)$ & $0 / 13(0.0)$ & .46 \\
\hline \multicolumn{5}{|l|}{$\mathrm{SAGE} \dagger$} \\
\hline Baseline & $2.0(0.0,3.5)$ & $2.0(0.0,5.0)$ & $0.5(0.0,2.0)$ & .02 \\
\hline \multicolumn{5}{|c|}{ Change from baseline } \\
\hline To $30 \mathrm{~d}$ & $0.0(-3.0,0.0)(0)$ & $-2.0(-3.0,0.0)(-100)$ & $0.0(-1.0,1.0)(0)$ & .20 \\
\hline To $90 \mathrm{~d}$ & $0.0(-3.0,0.0)(0)$ & $-0.5(-3.5,0.0)(-25)$ & $0.0(-2.0,0.0)(0)$ & .15 \\
\hline
\end{tabular}

Values are presented as mean \pm standard deviation, mean \pm standard deviation ( $\%$ change from baseline), median (quartile 1 , quartile 3$)(\%$ change from baseline $)$, or $\mathrm{n} / \mathrm{N}(\%)$, Total numbers are included when they differ from those in the overall study group. MoCA, Montreal Cognitive Assessment; DSST, Digit Symbol Substitution Test; $S A G E$, Standard Assessment of Global Activities in the Elderly. *For both MoCA and DSST, a positive change from baseline represents an improvement in test score (cognitive improvement), whereas negative change represents a decline in test score (cognitive decline). $\dagger$ For SAGE, a negative change from baseline represents an improvement in test score (functional improvement), whereas positive change represents a decline in test score (functional decline). 
TABLE 3. Assessment completion rates

\begin{tabular}{|c|c|c|c|c|}
\hline Assessment & $\begin{array}{c}\text { Overall } \\
(N=49)\end{array}$ & $\begin{array}{c}\text { No covert } \\
\text { stroke } \\
(n=30)\end{array}$ & $\begin{array}{c}\text { Covert } \\
\text { stroke } \\
(\mathrm{n}=19)\end{array}$ & $P$ value \\
\hline \multicolumn{5}{|l|}{ MoCA } \\
\hline Baseline & $46(94)$ & $29(97)$ & $17(89)$ & .31 \\
\hline \multicolumn{5}{|c|}{ Change from baseline } \\
\hline To discharge & $40(82)$ & $27(90)$ & $13(68)$ & .06 \\
\hline To $30 \mathrm{~d}$ & $37(76)$ & $24(80)$ & $13(68)$ & .36 \\
\hline \multicolumn{5}{|l|}{ DSST } \\
\hline Baseline & $47(96)$ & $30(100)$ & $17(89)$ & .07 \\
\hline \multicolumn{5}{|c|}{ Change from baseline } \\
\hline To discharge & $42(86)$ & $29(97)$ & $13(68)$ & .006 \\
\hline To $30 \mathrm{~d}$ & $38(78)$ & $25(83)$ & $13(68)$ & .22 \\
\hline \multicolumn{5}{|l|}{ SAGE } \\
\hline Baseline & $48(98)$ & $30(100)$ & $18(95)$ & .20 \\
\hline \multicolumn{5}{|c|}{ Change from baseline } \\
\hline To $30 \mathrm{~d}$ & $46(94)$ & $28(93)$ & $18(95)$ & .84 \\
\hline To $90 \mathrm{~d}$ & $45(92)$ & $28(93)$ & $17(89)$ & .63 \\
\hline
\end{tabular}

Values are presented as n (\%). MoCA, Montreal Cognitive Assessment; DSST, Digit Symbol Substitution Test; SAGE, Standard Assessment of Global Activities in the Elderly.

who had covert stoke followed by a clinical stroke 10 days later.

Patients with covert stroke may have an increased demand of health care resources. Median length of in-hospital stay was 2.5 days longer in patients who had a covert stroke (10.0 days; IQR, 6.0-14.0 days) compared with patients who did not have a covert stroke (7.5 days; IQR, 6.0-10.0 days). In addition, 17 (90\%) patients with covert stroke were discharged home from the hospital compared to $29(97 \%)$ patients without covert stroke.

\section{DISCUSSION}

This study demonstrates the feasibility of conducting a large cohort study to clearly establish the potential association between covert stroke and cognitive decline in patients 1 year after cardiac surgery and to determine whether surgical techniques that aim to reduce or avoid aortic manipulation can reduce rates of covert stroke. The primary outcome of NeuroVISION Cardiac pilot was feasibility. We demonstrated feasibility of DW-MRI scanning 3 to 9 days after CABG surgery at a rate of 1 patient per week. At our center, we were limited to 1 brain MRI series for the purpose of research per week due to limited availability of the MRI scanner. In the main study, we will recruit from multiple collaborating centers to overcome the limited MRI availability at our center. In addition, no patients were lost to follow-up at the final 90-day visit. Together, these results demonstrate good feasibility of the study protocol that will be used to inform the main study (Video 1).

We found a high incidence of perioperative covert stroke $(39 \%)$, which was more than 6-fold higher than the

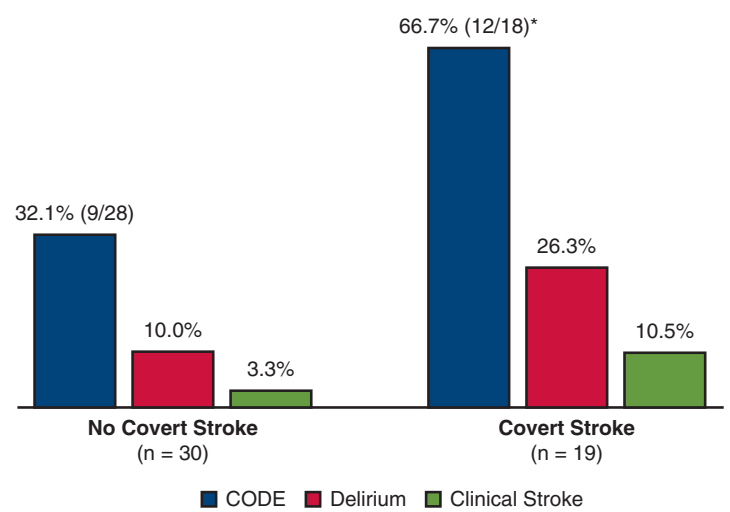

FIGURE 2. Shown left to right is the incidence of intraoperative cerebral oxygen desaturation events (blue bars), postoperative delirium (red bars), and clinical stroke within 30 days of coronary artery bypass grafting (CABG) surgery (green bars) in patients not experiencing (left) or experiencing (right) a perioperative covert stroke. Cerebral oxygen desaturation events ( $C O D E$ s) occurred when regional cerebral oxygen saturation (ie, oximetry) values declined $\geq 20 \%$ below baseline in either left, right, or both hemispheres during surgery. Three patients were excluded from the oximetry analysis: 2 patients with incomplete transcripts and 1 patient undergoing off-pump CABG surgery. *Covert stroke was associated with higher rates of CODEs during surgery (12 [67\%] patients experiencing covert stroke vs $9[32 \%]$ without covert stroke $[P=.02])$.

incidence of clinical stroke $(6 \%)$ in patients undergoing CABG surgery. Similar differences between covert stroke and clinical stroke rates have been reported in other small studies (covert stroke vs clinical stroke [fold change]): $16 \%$ versus $2 \%$ [8-fold] in a study of 50 patients ${ }^{22} ; 28 \%$ versus $3 \%$ [9-fold] in a study of 127 patients $^{23}$; and $20 \%$ versus $2 \%$ [10-fold] in a study of 104 patients. $^{24}$ The relatively high incidence of covert stroke and clinical stroke in our study may, in part, be explained by the relatively high mean age (67 years vs 61 years $^{23}$ or 69 years $^{24}$ ) of our cohort, as age is well-known risk factor for covert stroke. ${ }^{5}$ Our cohort also had at least 1 risk factor necessary for inclusion and thus would be expected to have an elevated risk of covert stroke compared with the other studies.

We also found high rates of clinical stroke in patients who had a covert stroke compared with patients who did not have a covert stroke $(11 \%$ vs $3 \%)$. Large population-based studies $^{9-12}$ and the large NeuroVISION ${ }^{7}$ study of noncardiac surgery patients suggest there is an association between covert stroke and future clinical stroke. These results are consistent with our findings and suggest that covert stroke increases the risk of future clinical stroke. If so covert stroke may serve as a valuable marker for predicting/preventing clinical stroke. The main NeuroVISION Cardiac study will definitively establish whether or not this association exists in the cardiac surgery setting.

The NeuroVISION Cardiac pilot study is the first study to examine the relationship between covert stroke and postoperative delirium in patients undergoing isolated 
TABLE 4. Clinical outcomes and adverse events

\begin{tabular}{|c|c|c|c|c|}
\hline Outcome & $\begin{array}{c}\text { Overall } \\
(N=49)\end{array}$ & $\begin{array}{l}\text { No covert stroke } \\
(\mathbf{n}=\mathbf{3 0})\end{array}$ & $\begin{array}{l}\text { Covert stroke } \\
\quad(\mathbf{n}=19)\end{array}$ & $\begin{array}{c}P \\
\text { value }\end{array}$ \\
\hline \multicolumn{5}{|l|}{ Perioperative } \\
\hline Delirium & $8(16.3)$ & $3(10.0)$ & $5(26.3)$ & .13 \\
\hline Delirium burden & $0.0(0.0-0.0)$ & $0.0(0.0-0.0)$ & $0.0(0.0-1.0)$ & .26 \\
\hline Cerebral oxygen desaturation event* & $21 / 46(45.7)$ & $9 / 28(32.1)$ & $12 / 18(66.7)$ & .02 \\
\hline \multicolumn{5}{|l|}{ Adverse events at $30 \mathrm{~d}$} \\
\hline Composite of death, MI, clinical stroke, and new renal failure requiring dialysis & $3(6.1)$ & $1(3.3)$ & $2(10.5)$ & - \\
\hline Death (all-causes) & $0(0.0)$ & $0(0.0)$ & $0(0.0)$ & - \\
\hline MI & $0(0.0)$ & $0(0.0)$ & $0(0.0)$ & - \\
\hline Clinical stroke & $3(6.1)$ & $1(3.3)$ & $2(10.5)$ & - \\
\hline New renal failure requiring dialysis & $0(0.0)$ & $0(0.0)$ & $0(0.0)$ & - \\
\hline \multicolumn{5}{|l|}{ Adverse events at $90 \mathrm{~d}$} \\
\hline Composite of death, MI, clinical stroke, and new renal failure requiring dialysis & $3(6.1)$ & $1(3.3)$ & $2(10.5)$ & - \\
\hline Death (all causes) & $1(2.0)$ & $0(0.0)$ & $1(5.3)$ & - \\
\hline MI & $0(0.0)$ & $0(0.0)$ & $0(0.0)$ & - \\
\hline Clinical stroke & $3(6.1)$ & $1(3.3)$ & $2(10.5)$ & - \\
\hline New renal failure requiring dialysis & $0(0.0)$ & $0(0.0)$ & $0(0.0)$ & - \\
\hline \multicolumn{5}{|l|}{ Use of health care resources } \\
\hline ICU length of stay (d) & $1.0(1.0-2.0)$ & $1.0(1.0-2.0)$ & $1.0(1.0-2.0)$ & .85 \\
\hline Hospital length of stay (d) & $9.0(6.0-11.0)$ & $7.5(6.0-10.0)$ & $10.0(6.0-14.0)$ & .12 \\
\hline Patients discharged to home & $46(93.9)$ & $29(96.7)$ & $17(89.5)$ & .31 \\
\hline
\end{tabular}

Values are presented as mean \pm standard deviation, $\mathrm{n}(\%), \mathrm{n} / \mathrm{N}(\%)$, or median (quartile 1-quartile 3). Total numbers are included when they differ from those in the overall study group. $M I$, Myocardial infarction; $I C U$, intensive care unit. *Three patients were excluded from the cerebral oxygen desaturation analysis: 2 patients with incomplete transcripts and 1 patient undergoing off-pump coronary artery bypass graft surgery.

CABG surgery. The overall incidence of delirium was $16 \%$, with high rates of delirium in patients who had a covert stroke compared with patients who did not have a covert stroke $(26 \%$ vs $10 \%))^{7}$ A similar result was found in a secondary analysis of a randomized trial with brain DW-MRI, where evidence of new ischemic lesions was found in 18 out of $28(64 \%)$ patients who had postoperative delirium and in 26 out of $51(51 \%)$ patients who did not have delirium. ${ }^{25}$ Our results are also consistent with recent findings from the NeuroVISION study, which demonstrated that perioperative covert stroke was associated with an increased risk of perioperative delirium in patients undergoing noncardiac surgery (hazard ratio, 2.24; 95\% CI, 1.06-4.73; absolute

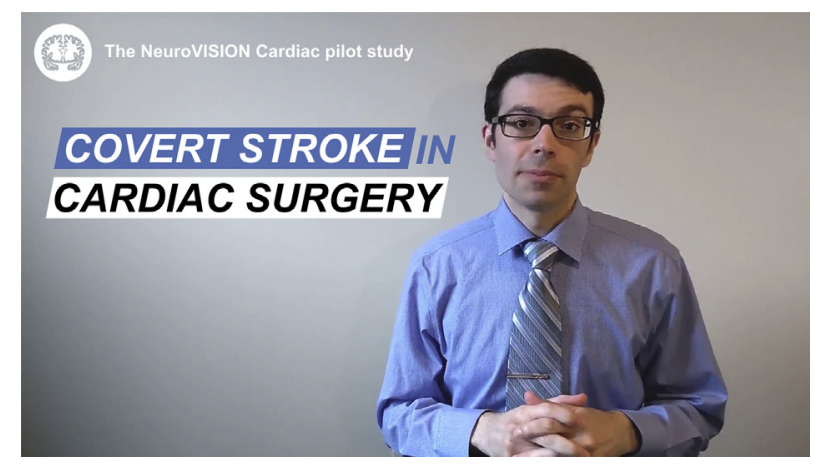

VIDEO 1. A video summary of the main results, relevance, and insights gained from the study. Video available at: https://www.jtcvs.org/article/ S2666-2736(20)30081-4/fulltext. risk increase $6 \% ; P=.03$ ). Together, these results support the hypothesis that covert stroke is associated with delirium after cardiac surgery. Future work should evaluate surgical techniques that aim to minimize aortic manipulation, such as the anaortic off-pump coronary artery bypass technique because this technique shows promise for decreasing the incidence of postoperative delirium (odds ratio, 0.26; $95 \% \mathrm{CI}, 0.09-0.68 ; P=.002)$ and early cognitive decline (odds ratio, $0.20 ; 95 \% \mathrm{CI}, 0.06-0.58 ; P<.001$ ) compared with the traditional off-pump technique. ${ }^{26}$

We showed that covert stroke is associated with higher rates of cerebral oxygen desaturation events during CABG surgery ( $67 \%$ vs $32 \%)$. A recent study of brain oximetry and DW-MRI in cardiac surgery patients found no difference in cerebral oxygen desaturation events between patients who had evidence of a DW-MRI lesion (44 [54\%]) and patients who did not have evidence of a DWMRI lesion $(30[43 \%]) .{ }^{27}$ In a separate analysis of the oximetry data we examined outcomes by intraoperative decline in oxygen saturation. Among 19 patients with covert stroke, $10(44 \%)$ patients had a cerebral oxygen desaturation event and $9(35 \%)$ did not have a cerebral oxygen desaturation event. ${ }^{28}$ Together, these results suggest that covert stokes (ie, cerebral ischemia) may be associated with acute declines in brain oxygenation (ie, cerebral hypoxemia).

Our study has several strengths. NeuroVISION Cardiac pilot has provided data to inform sample size calculations, 
Consequences of Perioperative Covert Stroke for CABG Surgery Patients

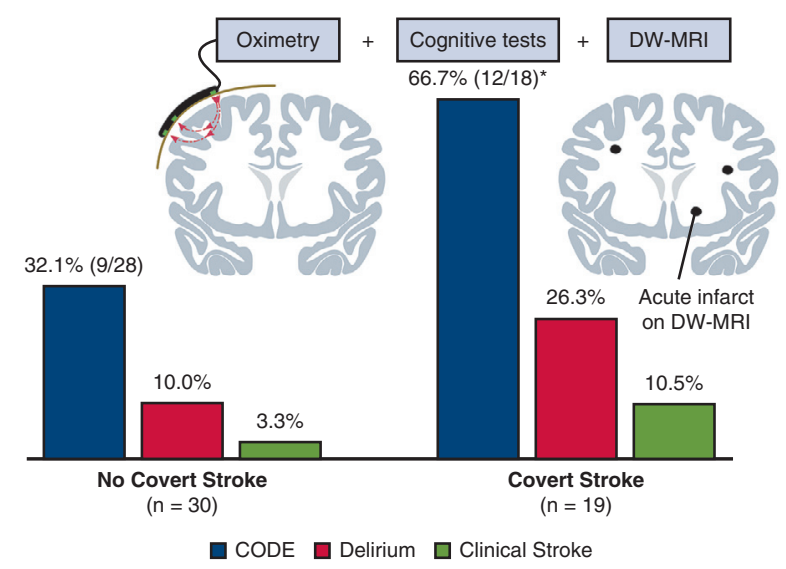

The incidence of perioperative covert stroke was 39\% (19/49 patients, $95 \% \mathrm{Cl} 26-53 \%$ ). This pilot study established the feasibility of conducting a large prospective study of the determinants and consequences of covert stroke in CABG surgery patients.

FIGURE 3. Shown left to right is the incidence of intraoperative cerebral oxygen desaturation events (blue bars), postoperative delirium (red bars) and clinical stroke within 30 days of coronary artery bypass grafting $(C A B G)$ surgery (green bars) in patients not experiencing (left) or experiencing (right) a perioperative covert stroke. Cerebral oxygen desaturation events (CODEs) occurred when regional cerebral oxygen saturation (ie, oximetry) values declined $\geq 20 \%$ below baseline in either left, right, or both hemispheres during surgery. Cognitive tests were administered before surgery, at hospital discharge and again 30 days after surgery. Acute infarcts (visible on diffusion-weighted magnetic resonance imaging $[D W-M R I]$ sequences) were present only in the brain (schematic diagrams in grey) of patients who experienced a perioperative covert stroke (black dots within the right brain diagram). Brain diagrams (frontal sections only) are not drawn to scale. Three patients were excluded from the oximetry analysis: 2 patients with incomplete transcripts and 1 patient undergoing off-pump CABG surgery. CI, Confidence interval; *Covert stroke was associated with higher rates of CODEs during surgery (12 [67\%] patients experiencing covert stroke vs $9[32 \%]$ without covert stroke $[P=.02])$.

number of sites required, and expected costs for a larger cohort study of the association between covert stroke and cognitive decline. This study is among the first to examine the relationship between covert stroke and postoperative delirium in patients undergoing isolated CABG surgery to report an association between covert stroke and acute declines in regional cerebral oxygen saturation (Figure 3).

\section{Limitations}

Our study also has several limitations. The single-center pilot study design has limited generalizability and was not powered to inform the clinical or cognitive influence of covert stroke. In this regard, the $P$ values provided should be interpreted with caution. For logistical reasons, followup was limited to 30 days. Cognitive assessment scores often improve moderately (Cohen's $d, 0.36-1.19$ ) with repeat testing over short intervals (within 90 days) in adults due to the practice effect. ${ }^{28,29}$ In the full-scale study, we intend to extend the follow-up to 1 year after surgery to minimize practice effects and to evaluate the long-term consequences of covert stroke because several studies suggest that deficits in cognitive function can persist up to 1 year after cardiac surgery. ${ }^{2,6}$ Twelve $(24 \%)$ patients did not complete both the baseline and 30-day MoCA limiting the analysis of changes in cognitive function. To minimize systematic bias from incomplete MoCA assessments we will collect the reasons for missing data and will use a method of evidence-informed data imputation in the larger study.

\section{CONCLUSIONS}

There is growing interest in determining the causes and consequences of covert strokes, especially in cardiac surgery patients who are at high risk for neurological complications. This pilot study established the feasibility of conducting a large prospective cohort study of covert stroke in CABG surgery patients and suggests that more than 1 in 3 patients aged $67 \pm 9$ years will experience a perioperative covert stroke. A larger study is needed now to clearly establish an association between covert stoke and cognitive decline 1 year after surgery and determine whether surgical techniques that aim to reduce or avoid aortic manipulation can reduce rates and consequences of covert stroke.

\section{Conflict of Interest Statement}

Medtronic provided access to a NIRS monitor for the duration of the study. Dr Mukul Sharma declares financial relationships with Bayer and Bristol Myers Squibb. Funding was received from Regional Medical Associates in the form of a Resident Research grant. All other authors reported no conflicts of interest.

The Journal policy requires editors and reviewers to disclose conflicts of interest and to decline handling or reviewing manuscripts for which they may have a conflict of interest. The editors and reviewers of this article have no conflicts of interest.

\section{References}

1. Yusuf S, Zucker D, Passamani E, Fisher LD, Takaro T, Kennedy JW, et al. Effect of coronary artery bypass graft surgery on survival: overview of 10-year results from randomised trials by the coronary artery bypass graft surgery trialists collaboration. Lancet. 1994;344:563-70.

2. Newman MF, Kirchner JL, Phillips-Bute B, Gaver V, Grocott H, Jones RH, et al. Longitudinal assessment of neurocognitive function after coronary-artery bypass surgery. N Engl J Med. 2001;344:395-402.

3. Seco M, Edelman JJB, Van Boxtel B, Forrest P, Byrom MJ, Wilson MK, et al. Neurologic injury and protection in adult cardiac and aortic surgery. $J$ Cardiothorac Vasc Anesth. 2015;29:185-95.

4. Selnes OA, Gottesman RF, Grega MA, Baumgartner WA, Zeger SL, McKhann GM. Cognitive and neurologic outcomes after coronary-artery bypass surgery. N Engl J Med. 2012;366:250-7.

5. Vermeer SE, Longstreth WT Jr, Koudstaal PJ. Silent brain infarcts: a systematic review. Lancet Neurol. 2007;6:611-9.

6. Saczynski JS, Marcantonio ER, Quach L, Fong TG, Gross A, Inouye SK, et al. Cognitive trajectories after postoperative delirium. N Engl J Med. 2012;367: 30-9.

7. NeuroVISION Investigators, Mrkobrada M, Chan MT, Cowan D, Campbell D, Wang CY, Torres D, et al. Perioperative covert stroke in patients undergoing 
non-cardiac surgery (NeuroVISION): a prospective cohort study. Lancet. 2019; 394:1022-9.

8. Vermeer SE, Prins ND, den Heijer T, Hofman A, Koudstaal PJ, Breteler MM. Silent brain infarcts and the risk of dementia and cognitive decline. $N$ Engl J Med. 2003;348:1215-22.

9. Vermeer SE, Hollander M, van Dijk EJ, Hofman A, Koudstaal PJ, Breteler MM. Silent brain infarcts and white matter lesions increase stroke risk in the general population: the Rotterdam scan study. Stroke. 2003;34:1126-9.

10. Bernick C, Kuller L, Dulberg C, Longstreth WT Jr, Manolio T, Beauchamp N, et al. Silent MRI infarcts and the risk of future stroke: the cardiovascular health study. Neurology. 2001;57:1222-9.

11. Debette S, Beiser A, DeCarli C, Au R, Himali JJ, Kelly-Hayes M, et al. Association of MRI markers of vascular brain injury with incident stroke, mild cognitive impairment, dementia, and mortality: the Framingham offspring study. Stroke. 2010;41:600-6.

12. Windham BG, Deere B, Griswold ME, Wang W, Bezerra DC, Shibata D, et al. Small brain lesions and incident stroke and mortality: a cohort study. Ann Intern Med. 2015;163:22-31.

13. Wolman RL, Nussmeier NA, Aggarwal A, Kanchuger MS, Roach GW, Newman MF, et al. Cerebral injury after cardiac surgery: identification of a group at extraordinary risk. Stroke. 1999;30:514-22.

14. Roach GW, Kanchuger M, Mangano CM, Newman M, Nussmeier N, Wolman R, et al. Adverse cerebral outcomes after coronary bypass surgery. $N$ Engl J Med. 1996;335:1857-64

15. Indja B, Woldendorp K, Vallely MP, Grieve SM. Silent brain infarcts following cardiac procedures: a systematic review and meta-analysis. J Am Heart Assoc. 2019;8:e010920.

16. Zheng F, Sheinberg R, Yee MS, Ono M, Zheng Y, Hogue CW. Cerebral nearinfrared spectroscopy (NIRS) monitoring and neurologic outcomes in adult cardiac surgery patients and neurologic outcomes: a systematic review. Anesth Analg. 2013;116:663-76.

17. Nasreddine ZS, Phillips NA, Bédirian V, Charbonneau S, Whitehead V, Collin I, et al. The Montreal cognitive assessment, MoCA: a brief screening tool for mild cognitive impairment. J Am Geriatr Soc. 2005;53:695-9.

18. Salthouse TA. The role of memory in the age decline in digit-symbol substitution performance. J Gerontol. 1978;33:232-8.

19. Jaeger J. Digit symbol substitution test: the case for sensitivity over specificity in neuropsychological testing. J Clin Psychopharmacol. 2018;38:513.
20. Inouye SK, van Dyck CH, Alessi CA, Balkin S, Siegal AP, Horwitz RI. Clarifying confusion: the confusion assessment method: a new method for detection of delirium. Ann Intern Med. 1990;113:941-8.

21. Bosch J, O’Donnell M, Swaminathan B, Lonn EM, Sharma M, Dagenais G, et al Effects of blood pressure and lipid lowering on cognition: results from the HOPE-3 study. Neurology. 2019;92:e1435-46.

22. Djaiani G, Fedorko L, Borger M, Mikulis D, Carroll J, Cheng D, et al. Mild to moderate atheromatous disease of the thoracic aorta and new ischemic brain lesions after conventional coronary artery bypass graft surgery. Stroke. 2004;35: e356-8.

23. Nah HW, Lee JW, Chung CH, Choo SJ, Kwon SU, Kim JS, et al. New brain in farcts on magnetic resonance imaging after coronary artery bypass graft surgery: lesion patterns, mechanism, and predictors. Ann Neurol. 2014;76:347-55.

24. Tachibana H, Hiraoka A, Saito K, Naito Y, Chikazawa G, Tamura K, et al. Incidence and impact of silent brain lesions after coronary artery bypass grafting. $J$ Thorac Cardiovasc Surg. October 16, 2019 [Epub ahead of print].

25. Brown CH IV, Faigle R, Klinker L, Bahouth M, Max L, LaFlam A, et al. The association of brain MRI characteristics and postoperative delirium in cardiac surgery patients. Clin Ther. 2015;37:2686-99.

26. Szwed K, Pawliszak W, Szwed M, Tomaszewska M, Anisimowicz L, Borkowska A. Reducing delirium and cognitive dysfunction after off-pump coronary bypass: a randomized trial. J Thorac Cardiovasc Surg. October 1, 2019 [Epub ahead of print].

27. Holmgaard F, Vedel A, Langkilde A, Lange T, Nilsson J, Ravn H. Differences in regional cerebral oximetry during cardiac surgery for patients with or without postoperative cerebral ischaemic lesions evaluated by magnetic resonance imaging. Br J Anaesth. 2018;121:1203-11.

28. Murkin JM, Newman SP, Stump DA, Blumenthal JA. Statement of consensus on assessment of neurobehavioral outcomes after cardiac surgery. Ann Thorac Surg. 1995;59:1289-95.

29. Bartels C, Wegrzyn M, Wiedl A, Ackermann V, Ehrenreich H. Practice effects in healthy adults: a longitudinal study on frequent repetitive cognitive testing. $B M C$ Neurosci. 2010;11:118.

Key Words: stroke, covert, delirium, cognitive, coronary artery bypass graft surgery, $\mathrm{CABG}$, magnetic resonance imaging, MRI, oximetry 


\section{APPENDIX 1. STUDY QUESTIONNAIRES Assessments of Cognitive Decline}

The Montreal Cognitive Assessment (MoCA) is used extensively to measure cognitive decline after stroke and cerebrovascular disease. It has proved superior to the MiniMental State Exam, in assessments of language skills, visuo-spatial and executive function, and in the diagnosis of cognitive decline after stroke. ${ }^{1,2}$ The MoCA has shown good sensitivity and specificity for cognitive decline when compared with the full 60-minute National Institute of Neurological Disorders and Stroke battery of neuropsychological assessments 1 year after stroke. ${ }^{3}$ The MoCA instrument assesses multiple cognitive domains, and we believe this will provide a thorough assessment of the influence of acute perioperative covert stroke. MoCA scores range from 0 to 30 with higher a higher score indicating superior cognitive function. In the CABG Off- or On-Pump Revascularization Study (CORONARY) trial $(\mathrm{n}=2081)$ the average MoCA score was $23.2 \pm 4$ before coronary artery bypass graft (CABG) surgery. ${ }^{4}$ Average MoCA scores at preoperative baseline were similar $(24.2 \pm 3)$ in our NeuroVISION Cardiac pilot study. A decrease of 2 points or more in MoCA score is perceived to be important to patients, and has been used as a cutoff for important cognitive decline. ${ }^{5-8}$ In addition, patients with covert stroke are reported to have lower standardized mean MoCA scores compared with patients without covert stroke (standard mean difference -3.36 points; $95 \%$ confidence interval, -5.90 to -0.82 ; $P=.01)^{9}$

The Digit Symbol Substitution Test (DSST) is a common clinical test that is sensitive to cognitive decline and to changes in cognitive decline across a wide range of clinical populations. ${ }^{10}$ The DSST evaluates cognitive speed, attention, and executive functions by requiring participants to match symbols to numbers within a 120 second time limit using a key. Scores range from 0 to a maximum of 133 with a higher score indicating superior cognitive ability. In the CORONARY trial $(\mathrm{n}=1975)$ the average DSST score was $32.5 \pm 17.9$ before CABG surgery. ${ }^{4}$ In our NeuroVISION Cardiac pilot study the average DSST scores was $42.9 \pm 13$ before CABG surgery. A decrease of 5 or more points on the DSST is considered clinically meaningful cognitive decline on the basis of differences in score observed between patients aged 70 and 75 years in the Cardiovascular Health Study. ${ }^{8,11,12}$ We will use this threshold to identify patients with cognitive decline.

\section{Assessment of Global Function}

The Standard Assessment of Global-activities in the Elderly (SAGE) is a simple and brief 15 -item assessment of ability to independently carry out daily activities of independent living (ie, global function) in older adults. Points are awarded when some, moderate, or complete help is required to carry out a task. SAGE scores range from 0 to 45 with lower scores indicating superior physical function. In our NeuroVISION Cardiac pilot study the average SAGE scores was $2.4 \pm 2.7$ before CABG surgery. The test has recently (ie, 2019) proven useful in cognitive substudies of the large Heart Outcomes Prevention Evaluation-3 (HOPE-3) ${ }^{8}$ and Cardiovascular Outcomes for People Using Anticoagulation Strategies (COMPASS) ${ }^{13}$ trials.

\section{References}

1. Dong Y, Sharma VK, Chan BP-L, Venketasubramanian N, Teoh HL, Seet RC, et al. The Montreal cognitive assessment (MoCA) is superior to the Mini-Mental State Examination (MMSE) for the detection of vascular cognitive impairment after acute stroke. J Neurol Sci. 2010; 299:15-8.

2. Pendlebury ST, Cuthbertson FC, Welch SJ, Mehta Z, Rothwell PM. Underestimation of cognitive impairment by mini-mental state examination versus the Montreal cognitive assessment in patients with transient ischemic attack and stroke: a population-based study. Stroke. 2010;41:1290-3.

3. Pendlebury ST, Mariz J, Bull L, Mehta Z, Rothwell PM. MoCA, ACE-R, and MMSE versus the National Institute of Neurological Disorders and Stroke-Canadian Stroke Network vascular cognitive impairment harmonization standards neuropsychological battery after TIA and stroke. Stroke. 2012;43: 464-9.

4. Lamy A, Devereaux P, Prabhakaran D, Taggart DP, Hu S, Paolasso E, et al. Effects of off-pump and on-pump coronary-artery bypass grafting at 1 year. $N$ Engl J Med. 2013;368:1179-88.

5. Tan HH, Xu J, Teoh HL, Chan BP, Seet RC, Venketasubramanian N, et al. Decline in changing Montreal cognitive assessment (MoCA) scores is associated with post-stroke cognitive decline determined by a formal neuropsychological evaluation. PloS one. 2017;12:e173291.

6. Baracchini C, Mazzalai F, Gruppo M, Lorenzetti R, Ermani M, Ballotta E. Carotid endarterectomy protects elderly patients from cognitive decline: a prospective study. Surgery. 2012;151:99-106.

7. Mrkobrada M, Chan MT, Cowan D, Campbell D, Wang CY, Torres D, et al. Perioperative covert stroke in patients undergoing non-cardiac surgery (NeuroVISION): a prospective cohort study. Lancet. 2019;394:1022-9.

8. Bosch J, O’Donnell M, Swaminathan B, Lonn EM, Sharma M, Dagenais G, et al. Effects of blood pressure and lipid lowering on cognition: results from the HOPE-3 study. Neurology. 2019;92:e1435-46.

9. Lei C, Deng Q, Li H, Zhong L. Association between silent brain infarcts and cognitive function: a systematic review and meta-analysis. J Stroke Cerebrovasc Dis. 2019;28:2376-87.

10. Jaeger J. Digit symbol substitution test: the case for sensitivity over specificity in neuropsychological testing. J Clin Psychopharmacol. 2018;38:513.

11. Newman AB, Glynn NW, Taylor CA, Sebastiani P, Perls TT, Mayeux R, et al. Health and function of participants in the Long Life Family Study: a comparison with other cohorts. Aging (Albany NY). 2011;3:63.

12. Brumback T, Cao D, King A. Effects of alcohol on psychomotor performance and perceived impairment in heavy binge social drinkers. Drug Alcohol Depend. 2007;91:10-7.

13. Sharma M, Hart RG, Smith EE, Bosch J, Yuan F, Casanova A, et al. Rationale, design, and baseline participant characteristics in the MRI and cognitive substudy of the cardiovascular outcomes for people using anticoagulation strategies trial. Int J Stroke. 2019;14:270-81. 
APPENDIX TABLE 1. List of participating surgeons by enrollment rate

\begin{tabular}{lc}
\hline \multicolumn{1}{c}{ Name } & No. of patients enrolled \\
\hline André Lamy & 27 \\
Lloyd Semelhago & 17 \\
Adel Dyub & 11 \\
Dominic Parry & 5 \\
Victor Chu & 2 \\
Irene Cybuslky & 2 \\
Jee-Yong (John) Lee & 2 \\
Total & 66 \\
\hline
\end{tabular}

APPENDIX TABLE 2. List of study personnel by role

\begin{tabular}{ll}
\hline \multicolumn{1}{c}{ Name } & \multicolumn{1}{c}{ Role } \\
\hline Peter Koh & Biometrics programmer \\
Marko Mrkobrada & Co-investigator \\
\hline PJ Devereaux & Co-investigator \\
\hline Richard Whitlock & Co-investigator \\
\hline Yannick LeManach & Co-investigator \\
\hline Jessica Spence & Co-investigator/project \\
& officer \\
\hline Lisa Trombetta & Data management \\
\hline Mukul Sharma & Neurologist \\
\hline Sara Hussain & PhD student \\
\hline André Lamy & Principal investigator \\
\hline Jessica Vincent & Project manager \\
\hline Shirley Pettit (former) & Project manager \\
\hline Shauna Kennedy & Radiologist \\
\hline Austin Browne & Research assistant \\
\hline Patricia Power & Research assistant \\
\hline Sarah Apolcer & Research assistant \\
\hline Tracy Boland & Research assistant \\
\hline Ingrid Copland & Research coordinator \\
\hline Hyejung Jung (former) & Statistician \\
\hline Rajibul Mian & Statistician \\
\hline Amelia Trombetta & Undergraduate student \\
\hline Stephanie Gagnon & Undergraduate student \\
\hline & \\
\hline
\end{tabular}

